\title{
A MASSERA-TYPE CRITERION FOR ALMOST PERIODIC SOLUTIONS OF HIGHER-ORDER DELAY OR ADVANCE ABSTRACT FUNCTIONAL DIFFERENTIAL EQUATIONS
}

\author{
NGUYEN VAN MINH AND HA BINH MINH
}

Received 24 January 2003

Let $u$ be a given bounded uniformly continuous mild solution of a higher-order abstract functional differential equation of delay or advance type. We give a so-called Masseratype criterion for the existence of a mild solution, which is a "spectral component" of $u$ with spectrum similar to the one of the forcing term $f$. Various spectral criteria for the existence of almost periodic and quasiperiodic mild solutions are given.

\section{Introduction}

The asymptotic behavior of solutions of differential equations in Banach spaces has been extensively studied since the last two decades. One of the interesting topics in this study is to find conditions for the existence of almost periodic solutions. We refer the reader to $[1,2,5,6,8,9,10,11,12,13,14,15,16,17]$ for more information.

The equation which we consider in this paper is of the form

$$
A_{0} u(t)+\sum_{k=1}^{n} A_{k} u^{(k)}(t)+\sum_{l=0}^{m} \int_{a}^{b} d B_{l}(\eta) u^{(l)}(t+\eta)=f(t), \quad t \in \mathbb{R}
$$

where $A_{0}$ is a closed linear operator acting on a Banach space $\mathbb{X} ; A_{k} \in L(\mathbb{X})$, for all $k=1, \ldots, n ; B_{l} \in \operatorname{BV}([a, b], L(\mathbb{X}))$, for all $l=0, \ldots, m ; f$ is an $\mathbb{X}$-valued almost periodic function on $\mathbb{R}$.

The problem with which we are concerned is to find (spectral) conditions for the existence of an almost periodic mild solution of (1.1) with the same structure of spectrum as the one of $f$. Such conditions are commonly referred to as Massera-type criteria for the existence of solutions with special structure of spectrum (see, e.g., $[7,9,16]$ ).

There have been several methods so far to deal with this problem for different kinds of equations. Our method of study is to use the one in [9] combined with the notion of almost periodic spectrum of bounded functions. As a result, we obtain a new Massera-type criterion for the existence of almost periodic mild solutions (see Corollary 3.7) which improves several known ones in $[4,7,9]$. 


\section{Preliminaries}

2.1. Notation. Throughout the paper, $\mathbb{R}, \mathbb{C}, \mathbb{X}$ stand for the sets of real, complex numbers, and a complex Banach space, respectively; $L(\mathbb{X}), \mathrm{BC}(\mathbb{R}, \mathbb{X}), \mathrm{BUC}(\mathbb{R}, \mathbb{X}), \operatorname{AP}(\mathbb{X})$ denote the spaces of linear bounded operators on $\mathbb{X}$, all $\mathbb{X}$-valued bounded continuous, bounded uniformly continuous, and almost periodic functions in Bohr's sense (see [6, page 4]) with sup-norm, respectively; $\mathscr{S}$ denotes the Schwartz space of all functions $\varphi \in C^{\infty}(\mathbb{R})$ such that each derivative of $\varphi$ decays faster than any polynomial. $\operatorname{By} \operatorname{BV}([a, b]$, $L(\mathbb{X})$ ), we denote the space of all functions from $[a, b]$ into $L(\mathbb{X})$ which are of bounded variation. For a linear operator $A$, we denote by $D(A), \sigma(A)$ the domain and the spectrum of $A$. Let $(S(t))_{t \in \mathbb{R}}$ be the translation group on $\mathrm{BUC}(\mathbb{R}, \mathbb{X})$ given by

$$
(S(t) u)(s):=u(t+s), \quad \forall s, t \in \mathbb{R}, \forall u \in \operatorname{BUC}(\mathbb{R}, \mathbb{X})
$$

whose infinitesimal generator is $\mathscr{D}:=d / d t$, defined on $D(\mathscr{D}):=\mathrm{BUC}^{1}(\mathbb{R}, \mathbb{X})$ which consists of all functions $f \in \mathrm{BUC}(\mathbb{R}, \mathbb{X})$ such that the derivative $f^{\prime}$ exists as an element of $\operatorname{BUC}(\mathbb{R}, \mathbb{X})$. Similarly, we define the space $\mathrm{BUC}^{k}(\mathbb{R}, \mathbb{X})$ for every natural number $k$.

2.2. Spectral theory of functions. In this paper, $\operatorname{sp}(u)$ stands for the Beurling spectrum of a given bounded uniformly continuous function $u$, which is defined by

$$
\operatorname{sp}(u):=\left\{\xi \in \mathbb{R}: \forall \varepsilon>0, \exists \varphi \in L^{1}(\mathbb{R}): \operatorname{supp} \tilde{\varphi} \subset(\xi-\varepsilon, \xi+\varepsilon), \varphi * u \neq 0\right\},
$$

where

$$
\tilde{\varphi}(s):=\int_{-\infty}^{\infty} e^{- \text {ist }} f(t) d t, \quad \varphi * u(s):=\int_{-\infty}^{\infty} \varphi(s-t) u(t) d t
$$

In the case of $u \in \mathrm{BUC}(\mathbb{R}, \mathbb{X})$, the Beurling spectrum coincides with the Carleman spectrum which consists of all $\xi \in \mathbb{R}$ such that the Carleman-Fourier transform of $u$, defined by

$$
\hat{u}(\lambda):= \begin{cases}\int_{0}^{\infty} e^{-\lambda t} u(t) d t & (\operatorname{Re} \lambda>0), \\ -\int_{0}^{\infty} e^{\lambda t} u(-t) d t & (\operatorname{Re} \lambda<0)\end{cases}
$$

has no holomorphic extension to any neighborhood of $i \xi$ (see [11, Proposition 0.5 , page $22])$. In turn, the Beurling spectrum of $u$ coincides with its Arveson spectrum, defined by (see $[1$, Section 2])

$$
\operatorname{isp}(u)=\sigma\left(\mathscr{D}_{u}\right)
$$

where $\mathscr{D}_{u}$ is the infinitesimal generator of the restriction of the translation group $\left(\left.S(t)\right|_{\mathcal{M}_{u}}\right)_{t \in \mathbb{R}}$ to the closed subspace $\mathcal{M}_{u}:=\overline{\operatorname{span}\{S(\tau) u, \tau \in \mathbb{R}\}}$. 
Below we list some properties of the spectra of functions which we will need in the sequel.

Proposition 2.1. Let $u, u_{n}, v \in \operatorname{BUC}(\mathbb{R}, \mathbb{X})$ such that $\lim _{n \rightarrow \infty}\left\|u_{n}-u\right\|=0, \psi \in \mathscr{Y}$, and $\Lambda \subset \mathbb{R}$. Then

(i) $\operatorname{sp}(u)$ is closed;

(ii) $\operatorname{sp}(u+v) \subset \operatorname{sp}(u) \cup \operatorname{sp}(v)$;

(iii) $\operatorname{sp}(\psi * u) \subset \operatorname{sp}(u) \cap \operatorname{supp} \tilde{\psi}$;

(iv) $\operatorname{sp}(u-\psi * u) \subset \operatorname{sp}(u) \cap \operatorname{supp}(1-\tilde{\psi})$;

(v) if $\tilde{\psi} \equiv 1$ on a neighbourhood of $\operatorname{sp}(u)$ then $\psi * u=u$;

(vi) if $\operatorname{sp}(u) \cap \operatorname{supp} \tilde{\psi}=\varnothing$, then $\psi * u=0$;

(vii) if $\operatorname{sp}\left(u_{n}\right) \subset \Lambda$, for all $n$, then $\operatorname{sp}(u) \subset \bar{\Lambda}$;

(viii) if $\operatorname{sp}(u)$ is countable, and $\mathbb{X}$ does not contain any subspace which is isomorphic to the space of numerical null-sequences $c_{0}$, then $u$ is almost periodic;

(ix) if $u$ is uniformly continuous and $\operatorname{sp}(u)$ is discrete, then $u$ is almost periodic.

Proof. We refer the reader to [11, Propositions 0.4, 0.6, Theorem 0.8, pages 20-25] and [6, Chapter 6] for the proofs of (i)-(viii) and [3] for (ix).

Remark 2.2. The condition $\mathbb{X} \not \supset c_{0}$ in Proposition 2.1 (viii) can be replaced by one of the following conditions:

(a) $u(\mathbb{R})$ is relatively weakly compact in $\mathbb{X}$;

(b) $u$ is totally ergodic, that is, the limit

$$
M_{\eta} u=\lim _{\tau \rightarrow \infty} \frac{1}{2 \tau} \int_{-\tau}^{\tau} e^{i \eta s} S(s) u d s
$$

exists in $\operatorname{BUC}(\mathbb{R}, \mathbb{X})$, for all $\eta \in \mathbb{R}$.

For more information, we refer the reader to [6, page 92], [1, Section 3], and [15].

Now we recall that the almost periodic spectrum of a bounded uniformly continuous function $u$, (which will be called AP-spectrum for the sake of simplicity) is defined by

$$
\operatorname{sp}_{\mathrm{AP}}(u):=\left\{\xi \in \mathbb{R}: \forall \varepsilon>0, \exists \varphi \in L^{1}(R): \operatorname{supp} \tilde{\varphi} \subset(\xi-\varepsilon, \xi+\varepsilon), \varphi * u \notin \operatorname{AP}(\mathbb{X})\right\}
$$

To proceed, we recall some known facts in [1] about the notion of AP-spectrum. Let $Y:=\operatorname{BUC}(\mathbb{R}, \mathbb{X}) / \mathrm{AP}(\mathbb{X})$ and let $\pi: \operatorname{BUC}(\mathbb{R}, \mathbb{X}) \rightarrow Y$ be the quotient map. Since $\operatorname{AP}(\mathbb{X})$ is invariant with respect to the group $(S(t))_{t \in \mathbb{R}},(S(t))_{t \in \mathbb{R}}$ induces a strongly continuous group on $Y$, denoted by $(\bar{S}(t))_{t \in \mathbb{R}}$, which is defined by the formula

$$
\bar{S}(t) \pi(u):=\pi(S(t) u), \quad \forall t \in \mathbb{R}, \forall u \in \operatorname{BUC}(\mathbb{R}, \mathbb{X}) .
$$

Let $\tilde{\mathscr{D}}$ denote the infinitesimal generator of the group $(\bar{S}(t))_{t \in \mathbb{R}}($ see $[2])$.

Similarly to the Beurling spectrum of a function $u$, its AP-spectrum coincides with the Arveson spectrum of $\pi(u)$, that is,

$$
\operatorname{isp}_{\mathrm{AP}}(u)=\sigma\left(\tilde{\mathscr{D}}_{\pi(u)}\right),
$$


where $\tilde{\mathscr{D}}_{\pi(u)}$ is the infinitesimal generator of the restriction of the translation group $\left(\left.\bar{S}(t)\right|_{\mathcal{M}_{\pi(u)}}\right)_{t \in \mathbb{R}}$ to the subspace $\mathcal{M}_{\pi(u)}:=\overline{\operatorname{span}\{\bar{S}(\tau) \pi(u), \tau \in \mathbb{R}\}}$ (see [1] for details).

To verify when a point belongs to $\operatorname{sp}_{\mathrm{AP}}(u)$, we may use [1, Section 2, Lemma 2.2], which can be stated in our terminology as follows.

Lemma 2.3. Let $\pi(u) \in Y$ and $\eta \in \mathbb{R}$. Assume that $R(\lambda, \tilde{D}) \pi(u)(\operatorname{Re} \lambda>0)$ has a holomorphic extension to a neighborhood of i $\eta$. Then i $\notin \sigma\left(\tilde{D}_{\pi(u)}\right)$, and equivalently $\eta \notin \operatorname{sp}_{\mathrm{AP}}(u)$.

We list below some main properties of AP-spectra of functions.

Proposition 2.4. Let $u, v, u_{n} \in \operatorname{BUC}(\mathbb{R}, \mathbb{X})$ such that $\lim _{n \rightarrow \infty}\left\|u_{n}-u\right\|=0, \psi \in \mathscr{Y}$, and $\Lambda$ is a subset of $\mathbb{R}$. Then

(i) $\operatorname{sp}_{\mathrm{AP}}(u+v) \subset \operatorname{sp}_{\mathrm{AP}}(u) \cup \mathrm{sp}_{\mathrm{AP}}(v)$;

(ii) $\operatorname{sp}_{\mathrm{AP}}(\psi * u) \subset \operatorname{sp}_{\mathrm{AP}}(u) \cap \operatorname{supp} \tilde{\psi}$;

(iii) $\operatorname{sp}_{\mathrm{AP}}(u-\psi * u) \subset \operatorname{sp}_{\mathrm{AP}}(u) \cap \operatorname{supp}(1-\tilde{\psi})$;

(iv) if $\operatorname{sp}_{\mathrm{AP}}\left(u_{n}\right) \subset \Lambda$, for all $n$, then $\operatorname{sp}_{\mathrm{AP}}(u) \subset \bar{\Lambda}$;

(v) if $\operatorname{sp}_{\mathrm{AP}}(u)$ is countable, and $\mathbb{X}$ does not contain $c_{0}$, then $u$ is almost periodic.

Proof. (i)-(ii) Using two basic properties of almost periodic functions: $u+v \in \mathrm{AP}(\mathbb{X})$ and $\psi * u \in \mathrm{AP}(\mathbb{X})$ whenever $u, v \in \mathrm{AP}(\mathbb{X}), \psi \in \mathscr{Y}$, from the definition of AP-spectrum, we can verify that (i) holds and

$$
\operatorname{sp}_{\mathrm{AP}}(\psi * u) \subset \operatorname{sp}_{\mathrm{AP}}(u) .
$$

It remains to prove that $\operatorname{sp}_{\mathrm{AP}}(\psi * u) \subset \operatorname{supp} \tilde{\psi}$. In fact, by Proposition 2.1(iii), we have

$$
\operatorname{sp}_{\mathrm{AP}}(\psi * u) \subset \operatorname{sp}(\psi * u) \subset \operatorname{supp} \tilde{\psi},
$$

thus proving (ii).

(iii) By Proposition 2.1(iv), Proposition 2.4(i), and (2.10), we have

$$
\begin{gathered}
\operatorname{sp}_{\mathrm{AP}}(u-\psi * u) \subset \operatorname{sp}_{\mathrm{AP}}(u) \cup \operatorname{sp}_{\mathrm{AP}}(\psi * u) \subset \operatorname{sp}_{\mathrm{AP}}(u), \\
\operatorname{sp}_{\mathrm{AP}}(u-\psi * u) \subset \operatorname{sp}(u-\psi * u) \subset \operatorname{supp}(1-\tilde{\psi}) .
\end{gathered}
$$

Hence, $\operatorname{sp}_{\mathrm{AP}}(u-\psi * u) \subset \operatorname{sp}_{\mathrm{AP}}(u) \cap \operatorname{supp}(1-\tilde{\psi})$.

(iv) First, we will prove that $\operatorname{sp}_{\mathrm{AP}}(f) \subset \bar{\Lambda}$ if and only if $\varphi * f \in \operatorname{AP}(\mathbb{X})$ whenever $\varphi \in$ $L^{1}(\mathbb{R})$ and supp $\tilde{\varphi} \cap \bar{\Lambda}=\varnothing$. In fact, in what follows, we prove the "if" and "only if" parts.

"If" part. Suppose $\xi \notin \bar{\Lambda}$. Then, we show that $\xi \notin \operatorname{sp}_{\mathrm{AP}}(f)$. Since $\xi \notin \bar{\Lambda}$, there exists $\varepsilon>0$ such that $(\xi-\varepsilon, \xi+\varepsilon) \cap \bar{\Lambda}=\varnothing$. Therefore, by the assumption that for all $\varphi \in L^{1}(\mathbb{R})$ such that $\operatorname{supp} \tilde{\varphi} \subset(\xi-\varepsilon, \xi+\varepsilon)$, we have $\varphi * f \notin \operatorname{AP}(\mathbb{X})$, we get $\xi \notin \operatorname{sp}_{\mathrm{AP}}(f)$. This proves the "if" part.

"Only if" part. Suppose that $\operatorname{sp}_{\mathrm{AP}}(f) \subset \bar{\Lambda}$. We now show that for all $\varphi \in L^{1}(\mathbb{R}): \operatorname{supp} \tilde{\varphi}$ $\subset(\xi-\varepsilon, \xi+\varepsilon), \varphi * f \in \operatorname{AP}(\mathbb{X})$. Indeed, we have

$$
\operatorname{sp}_{\mathrm{AP}}(\varphi * f) \subset \operatorname{sp}_{\mathrm{AP}}(f) \cap \operatorname{supp} \tilde{\varphi} \subset \bar{\Lambda} \cap \operatorname{supp} \tilde{\varphi}=\oslash .
$$

Hence, $\varphi * f \in \operatorname{AP}(\mathbb{X})$. 
Now we prove (iv). From the above claim and by assumption, we have $\varphi * u_{n} \in \operatorname{AP}(\mathbb{X})$ whenever $\varphi \in L^{1}(\mathbb{R})$ and supp $\tilde{\varphi} \cap \bar{\Lambda}=\oslash$. On the other hand, since $\lim _{n \rightarrow \infty}\left\|u_{n}-u\right\|=0$, it follows that

$$
\mathrm{AP}(\mathbb{X}) \ni \varphi * u_{n} \longrightarrow \varphi * u
$$

as $n \rightarrow \infty$. Therefore, as $\operatorname{AP}(\mathbb{X})$ is closed, $\varphi * u \in \operatorname{AP}(\mathbb{X})$, that is, $\operatorname{sp}_{\mathrm{AP}}(u) \subset \bar{\Lambda}$.

(v) For the proof, the reader is referred to [6, page 92].

Remark 2.5. In analogy to Remark 2.2 , we can replace the condition $\mathbb{X} \not \supset c_{0}$ in Proposition 2.4(v) by one of conditions (a) or (b) in Remark 2.2. We refer the reader to [6, page 92], [1, Section 3], and [15] for more information.

Definition 2.6. A function $u \in \mathrm{BUC}(\mathbb{R}, \mathbb{X})$ is called a strong solution of (1.1) if $u(t) \in$ $D\left(A_{0}\right)$, for all $t \in \mathbb{R}$ and $u \in \operatorname{BUC}^{k}(\mathbb{R}, \mathbb{X})$, with $k=\max (m, n)$ and (1.1) holds for all $t \in \mathbb{R}$.

A function $u \in \operatorname{BUC}(\mathbb{R}, \mathbb{X})$ is a mild solution on $\mathbb{R}$ of $(1.1)$, if, for each $\psi \in L^{1}(\mathbb{R})$ such that its Fourier transform $\tilde{\psi}$ has compact support, the function $\psi * u$ is a strong solution of (1.1) with $f$ replaced by $\psi * f$.

Remark 2.7. (i) By the superposition principle, if $u$ is a mild solution of (1.1) and $\psi \in$ $L^{1}(\mathbb{R})$ has supp $\tilde{\psi}$ compact, then $u-\psi * u$ is also a mild solution of (1.1) with $f$ replaced by $f-\psi * f$.

(ii) In the case where $A_{0}$ generates a strongly continuous semigroup of linear operators and $A_{1}=-I, n=1, m=0$, the above-defined notion of mild solutions coincides with the usual one of mild solutions to inhomogeneous linear evolution equations, that is, bounded continuous solutions to the integral equation

$$
u(t)=T(t-s) u(s)+\int_{s}^{t} T(t-\xi) f(\xi) d \xi, \quad \forall t \geq s ; t, s \in \mathbb{R}
$$

where $(T(t))_{t \geq 0}$ is the semigroup generated by $A_{0}$. In the light of this, the above-defined notion of mild solutions can be regarded as a generalization of the usual one. There are several generalizations which can be found in $[8,10,11]$.

\section{Main results}

We begin this section with the notion of spectrum of (1.1), which is described as follows. Definition 3.1. For every $\lambda \in \mathbb{C}$, define an operator

$$
R(\lambda):=A_{0}+\sum_{k=1}^{n} \lambda^{k} A_{k}+\sum_{l=0}^{m} \lambda^{l} \int_{a}^{b} e^{\lambda \eta} d B_{l}(\eta)
$$


on $\mathbb{X}$ with $D(R(\lambda))=D\left(A_{0}\right)$, which is called a spectral operator of (1.1). The set

$$
\Sigma:=\{\lambda \in \mathbb{C}: R(\lambda) \text { is not invertible }\}
$$

is called the spectrum of (1.1).

In the case where (1.1) is of the form $\dot{x}=A_{0} x-f$, here $A_{0}$ is a linear closed operator, the spectral operator and the spectrum of (1.1), respectively, are

$$
R(\lambda)=\lambda-A_{0}, \quad \Sigma=\sigma\left(A_{0}\right)
$$

As is well known, $\Sigma$ is a closed subset of $\mathbb{C}$ and $R^{-1}(\lambda)$ is a holomorphic function from $\mathbb{C} \backslash \sigma\left(A_{0}\right)$ to $L(\mathbb{X})$. It is natural to ask if these properties hold true in the general case. The following lemma gives an answer to this question.

Lemma 3.2. Let $A_{0}$ be a linear closed operator on $\mathbb{X}, A_{k} \in L(\mathbb{X})$, for all $k=1, \ldots, n$, and $B_{l} \in \mathrm{BV}([a, b], L(\mathbb{X}))$, for all $l=0, \ldots, m$. Then

(i) $\Sigma$ is a closed set;

(ii) $R^{-1}: \mathbb{C} \backslash \Sigma \rightarrow L(\mathbb{X})$ is a holomorphic function.

Proof. (i) To prove the closedness of $\Sigma$, we will show that $\mathbb{C} \backslash \Sigma$ is open. For every $\lambda_{0} \in$ $\mathbb{C} \backslash \Sigma$, the operator $R^{-1}\left(\lambda_{0}\right)$ exists as an element of $L(\mathbb{X})$. Since $A_{0}$ is closed, $R^{-1}\left(\lambda_{0}\right)$ is a bounded operator by the closed graph theorem.

Setting

$$
G(\lambda):=\sum_{k=1}^{n} \lambda^{k} A_{k}+\sum_{l=0}^{m} \lambda^{l} \int_{a}^{b} e^{\lambda \eta} d B_{l}(\eta)
$$

we have

$$
R(\lambda) R^{-1}\left(\lambda_{0}\right)=I+\left[R(\lambda)-R\left(\lambda_{0}\right)\right] R^{-1}\left(\lambda_{0}\right)=I+\left[G(\lambda)-G\left(\lambda_{0}\right)\right] R^{-1}\left(\lambda_{0}\right) .
$$

It is easily seen that $\left[G(\lambda)-G\left(\lambda_{0}\right)\right] R^{-1}\left(\lambda_{0}\right) \in L(\mathbb{X})$, for all $\lambda \in \mathbb{C}$. Moreover, we have the estimate

$$
\begin{aligned}
\left\|\left[G(\lambda)-G\left(\lambda_{0}\right)\right] R^{-1}\left(\lambda_{0}\right)\right\| & \leq M\left\|G(\lambda)-G\left(\lambda_{0}\right)\right\| \\
& =M\left\|\sum_{k=1}^{n}\left(\lambda^{k}-\lambda_{0}^{k}\right) A_{k}+\sum_{l=0}^{m} \int_{a}^{b}\left(\lambda^{l} e^{\lambda \eta}-\lambda_{0}^{l} e^{\lambda_{0} \eta}\right) d B_{l}(\eta)\right\| \\
& \leq M \sum_{k=1}^{n}\left|\lambda^{k}-\lambda_{0}^{k}\right|\left\|A_{k}\right\|+M \sum_{l=0}^{m} \int_{a}^{b}\left|\lambda^{l} e^{\lambda \eta}-\lambda_{0}^{l} e^{\lambda_{0} \eta}\right| d V_{l}(\eta),
\end{aligned}
$$


where $M:=\left\|R^{-1}\left(\lambda_{0}\right)\right\| ; V_{l}(\eta):=\operatorname{Var}_{a}^{\eta}\left[B_{l}\right]$ is the variation of $B_{l}$ on the interval $[a, \eta]$. It follows that there exists a neighborhood $U_{\lambda_{0}}$ of $\lambda_{0}$ such that $\left\|\left[G(\lambda)-G\left(\lambda_{0}\right)\right] R^{-1}\left(\lambda_{0}\right)\right\|<1$, for all $\lambda \in U_{\lambda_{0}}$. This shows that there exists the bounded inverse operator

$$
\left[I+\left[G(\lambda)-G\left(\lambda_{0}\right)\right] R^{-1}\left(\lambda_{0}\right)\right]^{-1}, \quad \forall \lambda \in U_{\lambda_{0}}
$$

and hence, $R^{-1}(\lambda)$ exists in $U_{\lambda_{0}}$ and is given by the following formula:

$$
R^{-1}(\lambda)=R^{-1}\left(\lambda_{0}\right)\left[I+\left[G(\lambda)-G\left(\lambda_{0}\right)\right] R^{-1}\left(\lambda_{0}\right)\right]^{-1} .
$$

By the definition of $\Sigma$, we have $U_{\lambda_{0}} \subset \mathbb{C} \backslash \Sigma$, so $\mathbb{C} \backslash \Sigma$ is an open subset of $\mathbb{C}$. This completes the proof of (i).

(ii) Assume that $\lambda_{0} \in \mathbb{C} \backslash \Sigma$. By (i), we have proved

$$
R^{-1}(\lambda)=R^{-1}\left(\lambda_{0}\right)\left[I+\left[G(\lambda)-G\left(\lambda_{0}\right)\right] R^{-1}\left(\lambda_{0}\right)\right]^{-1}, \quad \forall \lambda \in U_{\lambda_{0}} .
$$

It may be noted that $G(\lambda)$ is holomorphic on the whole complex plane. Therefore, $R^{-1}$ : $\mathbb{C} \backslash \Sigma \rightarrow L(\mathbb{X})$ is a holomorphic function.

Set

$$
\Lambda:=\{\xi \in \mathbb{R}: R(i \xi) \text { is not invertible }\}
$$

We are going to estimate the spectra of a bounded mild solution of (1.1) which will play an important role in proving the main results of the paper.

Lemma 3.3. Assume that $A_{0}$ is a linear closed operator on $\mathbb{X}, A_{k} \in L(\mathbb{X})$, for all $k=1, \ldots, n$, and $B_{l} \in \mathrm{BV}([a, b], L(\mathbb{X}))$, for all $l=0, \ldots, m$. Let $u \in \mathrm{BUC}(\mathbb{R}, \mathbb{X})$ be a mild solution of $(1.1)$. Then

(i) $\operatorname{sp}(u) \subset \Lambda \cup \operatorname{sp}(f)$;

(ii) $\operatorname{sp}_{\mathrm{AP}}(u) \subset \Lambda$ if $f \in \mathrm{AP}(\mathbb{X})$.

Proof. We first prove that if (i) and (ii) hold for every strong solution, then so do they for any mild solution. In fact, let $u \in \mathrm{BUC}(\mathbb{R}, \mathbb{X})$ be a mild solution of (1.1). Let $K_{n} \in L^{1}(\mathbb{R})$ be a sequence of functions such that

(i) $\operatorname{supp} \tilde{K}_{n}=[-n, n]$;

(ii) for every $g \in \operatorname{BUC}(\mathbb{R}, \mathbb{X})$, if we set $g_{n}=g * K_{n}$, then $\lim _{n \rightarrow \infty} g_{n}=g$.

We refer the reader to [6, pages 87-88] for the construction of such a sequence of functions $K_{n}$. Since $u \in \mathrm{BUC}(\mathbb{R}, \mathbb{X})$ is a mild solution of $(1.1)$, it follows that $K_{n} * u$ is a strong solution of (1.1) with $f$ replaced by $K_{n} * f$. By the assumption that (i) holds for strong solutions, we have

$$
\begin{aligned}
\operatorname{sp}\left(K_{n} * u\right) & \subset \Lambda \cup \operatorname{sp}\left(K_{n} * f\right) \\
& \subset \Lambda \cup\left(\operatorname{sp}(f) \cap \operatorname{supp} \tilde{K}_{n}\right) \\
& \subset \Lambda \cup \operatorname{sp}(f), \quad \forall n=1,2, \ldots
\end{aligned}
$$

This shows that (i) holds for any mild solution. 
Now, assuming that (ii) holds for strong solutions, we show that so does (ii) for mild solutions. By definition, it suffices to show that $K_{n} * f \in \operatorname{AP}(\mathbb{X})$. But this is obvious because $K_{n} \in L^{1}(\mathbb{R})$ and $f$ is an almost periodic function. Thus,

$$
\operatorname{sp}_{\mathrm{AP}}\left(K_{n} * u\right) \subset \Lambda, \quad \forall n=1,2, \ldots
$$

Since $K_{n} * u$ converges uniformly to $u$ and by Propositions 2.1(iv) and 2.4(i), we have

$$
\operatorname{sp}(u) \subset \Lambda \cup \operatorname{sp}(f), \quad \operatorname{sp}_{\mathrm{AP}}(u) \subset \Lambda,
$$

which proves our claim.

Now, it remains to prove (i) and (ii) for strong solutions.

(i) Suppose that $u$ is a strong solution of (1.1). For a fixed $\eta \in \mathbb{R}$, let $v(t):=u(t+\eta)$, $b_{l}(t):=\int_{a}^{b} d B_{l}(\eta) u^{(l)}(t+\eta)$, for all $l=0, \ldots, m, t \in \mathbb{R}$. Since $u$ is a strong solution, it follows that $u^{(k)}, k=0, \ldots, n$, and $v^{(l)}, b_{l}, l=0, \ldots, m$, are bounded functions. Therefore, their Fourier-Carleman transforms exist and can be easily computed. In fact, by induction, we have

$$
\widehat{u^{(k)}}(\lambda)=\lambda^{k} \hat{u}(\lambda)-\sum_{j=0}^{k-1} \lambda^{j} u^{(k-j-1)}(0), \quad \forall k=1, \ldots, n
$$

Similarly,

$$
\begin{aligned}
\widehat{v^{(l)}}(\lambda) & =\lambda^{l} \hat{v}(\lambda)-\sum_{j=0}^{l-1} \lambda^{j} v^{(l-j-1)}(0) \\
& =\lambda^{l} e^{\lambda \eta}\left(\hat{u}(\lambda)-\int_{0}^{\eta} e^{-\lambda t} u(t) d t\right)-\sum_{j=0}^{l-1} \lambda^{j} u^{(l-j-1)}(\eta), \quad \forall l=1, \ldots, m .
\end{aligned}
$$

Now, we compute the Fourier-Carleman transforms of $b_{l}$, for all $l=0, \ldots, m$. For $\operatorname{Re} \lambda>0$,

$$
\begin{aligned}
\hat{b_{l}}(\lambda) & =\int_{0}^{\infty} e^{-\lambda t}\left(\int_{a}^{b} d B_{l}(\eta) u^{(l)}(t+\eta)\right) d t \\
& =\int_{a}^{b} d B_{l}(\eta)\left(\int_{0}^{\infty} e^{-\lambda t} u^{(l)}(t+\eta) d t\right) \\
& =\int_{a}^{b} d B_{l}(\eta) \widehat{v}^{(l)}(\lambda), \quad \forall l=1, \ldots, m
\end{aligned}
$$

and for $\operatorname{Re} \lambda<0$,

$$
\begin{aligned}
\hat{b}_{l}(\lambda) & =-\int_{0}^{\infty} e^{\lambda t}\left(\int_{a}^{b} d B_{l}(\eta) u^{(l)}(-t+\eta)\right) d t \\
& =-\int_{a}^{b} d B_{l}(\eta)\left(\int_{0}^{\infty} e^{\lambda t} u^{(l)}(-t+\eta) d t\right) \\
& =\int_{a}^{b} d B_{l}(\eta) \widehat{v^{(l)}}(\lambda), \quad \forall l=1, \ldots, m .
\end{aligned}
$$


Finally, for $\operatorname{Re} \lambda \neq 0$,

$$
\begin{aligned}
\hat{b}_{l}(\lambda)= & \int_{a}^{b} d B_{l}(\eta) \widehat{v^{(l)}}(\lambda) \\
= & \lambda^{l} \int_{a}^{b} e^{\lambda \eta} d B_{l}(\eta) \hat{u}(\lambda)-\lambda^{l} \int_{a}^{b} d B_{l}(\eta)\left(e^{\lambda \eta} \int_{0}^{\eta} e^{-\lambda t} u(t) d t\right) \\
& -\sum_{j=0}^{l-1} \lambda^{j} \int_{a}^{b} e^{\lambda \eta} d B_{l}(\eta) u^{(l-j-1)}(\eta), \quad \forall l=1, \ldots, m .
\end{aligned}
$$

In the case of $l=0$,

$$
\widehat{b_{0}}(\lambda)=\int_{a}^{b} e^{\lambda \eta} d B_{0}(\eta) \hat{u}(\lambda)-\int_{a}^{b} d B_{0}(\eta)\left(e^{\lambda \eta} \int_{0}^{\eta} e^{-\lambda t} u(t) d t\right) .
$$

Taking Fourier-Carleman transform in (1.1), by (3.14), (3.15), (3.18), (3.19), we see that $\hat{u}(\lambda) \in D\left(A_{0}\right)$, for all $\lambda \in \mathbb{C}$, and

$$
\begin{aligned}
A_{0} \hat{u}(\lambda) & +\sum_{k=1}^{n} A_{k}\left[\lambda^{k} \hat{u}(\lambda)-\sum_{j=0}^{k-1} \lambda^{j} u^{(k-j-1)}(0)\right] \\
+ & {\left[\int_{a}^{b} e^{\lambda \eta} d B_{0}(\eta) \hat{u}(\lambda)-\int_{a}^{b} d B_{0}(\eta)\left(e^{\lambda \eta} \int_{0}^{\eta} e^{-\lambda t} u(t) d t\right)\right] } \\
+ & \sum_{l=1}^{m}\left[\lambda^{l} \int_{a}^{b} e^{\lambda \eta} d B_{l}(\eta) \hat{u}(\lambda)-\lambda^{l} \int_{a}^{b} d B_{l}(\eta)\left(e^{\lambda \eta} \int_{0}^{\eta} e^{-\lambda t} u(t) d t\right)\right. \\
& \left.-\sum_{j=0}^{l-1} \lambda^{j} \int_{a}^{b} e^{\lambda \eta} d B_{l}(\eta) u^{(l-j-1)}(\eta)\right]=\hat{f}(\lambda) .
\end{aligned}
$$

Therefore,

$$
\begin{gathered}
{\left[A_{0}+\sum_{k=1}^{n} \lambda^{k} A_{k}+\sum_{l=0}^{m} \lambda^{l} \int_{a}^{b} e^{\lambda \eta} d B_{l}(\eta)\right] \hat{u}(\lambda)-\sum_{l=0}^{m} \lambda^{l} \int_{a}^{b} d B_{l}(\eta)\left(e^{\lambda \eta} \int_{0}^{\eta} e^{-\lambda t} u(t) d t\right)} \\
-\sum_{k=1}^{n} \sum_{j=0}^{k-1} \lambda^{j} u^{(k-j-1)}(0)-\sum_{l=1}^{m} \sum_{j=0}^{l-1} \lambda^{j} \int_{a}^{b} e^{\lambda \eta} d B_{l}(\eta) u^{(l-j-1)}(\eta)=\hat{f}(\lambda) .
\end{gathered}
$$

Finally, we arrive at

$$
R(\lambda) \hat{u}(\lambda)-p(\lambda)=\hat{f}(\lambda)
$$

where

$$
\begin{aligned}
p(\lambda):= & \sum_{l=0}^{m} \lambda^{l} \int_{a}^{b} d B_{l}(\eta)\left(e^{\lambda \eta} \int_{0}^{\eta} e^{-\lambda t} u(t) d t\right) \\
& +\sum_{k=1}^{n} \sum_{j=0}^{k-1} \lambda^{j} A_{k} u^{(k-j-1)}(0)+\sum_{l=1}^{m} \sum_{j=0}^{l-1} \lambda^{j} \int_{a}^{b} e^{\lambda \eta} d B_{l}(\eta) u^{(l-j-1)}(\eta) .
\end{aligned}
$$


Take $\xi_{0} \in \mathbb{R}$ such that $\xi_{0} \notin(\Lambda \cup \operatorname{sp}(f))$, that is, $\xi_{0} \notin \Lambda$ and $\xi_{0} \notin \operatorname{sp}(f)$. Since $\xi_{0} \notin \Lambda$, by Lemma 3.2, it follows that $R^{-1}(\lambda)$ exists and is a holomorpic function in a neighborhood of $i \xi_{0}$. Thus,

$$
\hat{u}(\lambda)=R^{-1}(\lambda)[\hat{f}(\lambda)+p(\lambda)]
$$

On the other hand, since $\xi_{0} \notin \mathrm{sp}(f), \hat{f}(\lambda)$ has a holomorphic extension to a neighborhood of $i \xi_{0}$ and $p(\lambda)$ is a holomorphic function. Hence, from (3.24), we see that $\hat{u}(\lambda)$ also has a holomorphic extension to a neighboorhood of $i \xi_{0}$, that is, $\xi_{0} \notin \operatorname{sp}(u)$. This proves (i).

(ii) Suppose that $\xi_{0} \in \mathbb{R}$ such that $\xi_{0} \notin \Lambda$. We have to prove that $\xi_{0} \notin \operatorname{sp}_{\mathrm{AP}}(u)$. To this end, we apply $S(t)$ to both sides of (1.1). Since the derivation operator commutes with shift operators, we obtain

$$
\mathscr{A}_{0} S(t) u+\sum_{k=1}^{n} \mathscr{A}_{k}(S(t) u)^{(k)}+\sum_{l=0}^{m} \int_{a}^{b} d \mathscr{S}_{l}(\eta)(S(t+\eta) u)^{(l)}=S(t) f, \quad t \in \mathbb{R}
$$

where $\mathscr{A}_{k}, k=0, \ldots, n$, and $\mathscr{B}_{l}(\eta), \eta \in[a, b], l=0, \ldots, m$, are multiplication operators defined by the formulas

$$
\begin{aligned}
\left(\mathscr{A}_{k} u\right)(t) & =A_{k} u(t), \quad k=0, \ldots, n, \\
\left(\mathscr{B}_{l}(\eta) u\right)(t) & =B_{l}(\eta) u(t), \quad \eta \in[a, b], l=0, \ldots, m .
\end{aligned}
$$

It is easily seen that for the chosen $i \xi_{0}:=\lambda_{0}$, the operator of multiplication by $R(\lambda)$,

$$
\mathscr{R}\left(\lambda_{0}\right):=\mathscr{A}_{0}+\sum_{k=1}^{n} \lambda_{0}^{k} \mathscr{A}_{k}+\sum_{l=0}^{m} \lambda_{0}^{l} \int_{a}^{b} e^{\lambda_{0} \eta} d \mathscr{B}_{l}(\eta)
$$

is invertible as well. Let $U(t):=S(t) u, F(t):=S(t) f$, for all $t \in \mathbb{R}$. We can rewrite (3.25) as follows:

$$
\mathscr{A}_{0} U(t)+\sum_{k=1}^{n} \mathscr{A}_{k} U^{(k)}(t)+\sum_{l=0}^{m} \int_{a}^{b} d \mathscr{B}_{l}(\eta) U^{(l)}(t+\eta)=F(t), \quad t \in \mathbb{R} .
$$

As in the proof of (i), by taking Fourier-Carleman transforms in (3.28), we get

$$
\mathscr{R}(\lambda) \hat{U}(\lambda)-q(\lambda)=\hat{F}(\lambda)
$$

where

$$
\begin{aligned}
q(\lambda):= & \sum_{l=0}^{m} \lambda^{l} \int_{a}^{b} d \mathscr{B}_{l}(\eta)\left(e^{\lambda \eta} \int_{0}^{\eta} e^{-\lambda t} U(t) d t\right) \\
& +\sum_{k=1}^{n} \sum_{j=0}^{k-1} \lambda^{j} \mathscr{A}_{k} U^{(k-j-1)}(0)+\sum_{l=1}^{m} \sum_{j=0}^{l-1} \lambda^{j} \int_{a}^{b} e^{\lambda \eta} d \mathscr{B}_{l}(\eta) U^{(l-j-1)}(\eta) .
\end{aligned}
$$


By Lemma 2.3, it suffices to show that $R(\lambda, \tilde{\mathscr{D}}) \pi(u)(\operatorname{Re} \lambda>0)$ has a holomorphic extension to a neighborhood of $i \xi_{0}$. In fact, by Lemma 3.2, $R^{-1}(\lambda)$ exists as a holomorpic function in the neighborhood of $i \xi_{0}:=\lambda_{0}$. Thus, around $\lambda_{0}$, from (3.29),

$$
\hat{U}(\lambda)=\mathscr{R}^{-1}(\lambda)[\hat{F}(\lambda)+q(\lambda)] .
$$

For $\operatorname{Re} \lambda>0$, by integral representation of the resolvent formula, we get

$$
\hat{U}(\lambda)=\widehat{(S(\cdot) u)}(\lambda)=\int_{0}^{\infty} e^{-\lambda t} S(t) u d t=R(\lambda, \mathscr{D}) u
$$

and similarly, $\hat{F}(\lambda)=(\widehat{S(\cdot) f})(\lambda)=R(\lambda, \mathscr{D}) f$. Hence, around $\lambda_{0}$,

$$
R(\lambda, \mathscr{D}) u=\mathscr{R}^{-1}(\lambda) R(\lambda, \mathscr{D}) f+\mathscr{R}^{-1}(\lambda) q(\lambda), \quad \operatorname{Re} \lambda>0
$$

We have $\pi\left(\mathscr{R}^{-1}(\lambda) R(\lambda, \mathscr{D}) f\right)=0$, because $f \in \mathrm{AP}(\mathbb{X})$ and $\mathscr{R}^{-1}(\lambda), R(\lambda, \mathscr{D})$ are bounded operators for $\operatorname{Re} \lambda>0$ and around $\lambda_{0}$. Thus, by applying $\pi$ to the above formula, we obtain

$$
\pi(R(\lambda, \mathscr{D}) u)=\pi\left(\mathscr{R}^{-1}(\lambda) q(\lambda)\right)
$$

Hence,

$$
R(\lambda, \tilde{\mathscr{D}}) \pi(u)=\pi\left(\mathscr{R}^{-1}(\lambda) q(\lambda)\right), \quad \operatorname{Re} \lambda>0 .
$$

Since $\mathscr{R}^{-1}(\lambda)$ and $q(\lambda)$ are holomorpic in the neighborhood $U_{\xi_{0}}$ of $i \xi_{0}$, it follows that $R(\lambda, \tilde{D}) \pi(u)\left(\lambda \in U_{\xi_{0}}, \operatorname{Re} \lambda>0\right)$ has a holomorphic extension to $U_{\xi_{0}}$. This proves the lemma.

We are in a position to state one of the main results of this paper.

Theorem 3.4. Let $A_{0}$ be a linear closed operator on $\mathbb{X}, A_{k} \in L(\mathbb{X})$, for all $k=1, \ldots, n$, and let $B_{l} \in \operatorname{BV}([a, b], L(\mathbb{X}))$, for all $l=0, \ldots, m$. Assume further that $\Lambda$ is bounded and $\operatorname{dist}(\Lambda \backslash \operatorname{sp}(f), \operatorname{sp}(f))>0$. Then, whenever equation (1.1) has a mild solution $u \in \mathrm{BUC}(\mathbb{R}, \mathbb{X})$, it has a mild solution $w \in \mathrm{BUC}(\mathbb{R}, \mathbb{X})$ such that

$$
\operatorname{sp}(w) \subset \operatorname{sp}(f)
$$

Proof. Set $d=\operatorname{dist}(\Lambda \backslash \operatorname{sp}(f), \operatorname{sp}(f))$. Since $\Lambda$ is compact, we can choose a continuous function $\varphi \in L^{1}(\mathbb{R})$ such that

$$
\begin{gathered}
\tilde{\varphi}=1 \quad \text { on } \Lambda \backslash \operatorname{sp}(f), \\
\operatorname{supp} \tilde{\varphi} \subset\left\{\lambda \in \mathbb{R}: \operatorname{dist}(\lambda, \Lambda \backslash \operatorname{sp}(f))<\frac{d}{2}\right\} .
\end{gathered}
$$

Set $w=u-\varphi * u$. According to Remark 2.7(i), the function $w$ is a mild solution of (1.1) with forcing term $f-\varphi * f$. Since $\operatorname{supp} \tilde{\varphi} \cap \operatorname{sp}(f)=\varnothing$, by Proposition 2.1(v), we get $f-\varphi * f=f$. Hence, $w$ is a mild solution of (1.1). 
Next, we will show that $\operatorname{sp}(w) \subset \mathrm{sp}(f)$. Indeed, by Proposition 2.1(iv) and Lemma 3.3(i), we have

$$
\begin{aligned}
\operatorname{sp}(w) & =\operatorname{sp}(u-\varphi * u) \\
& \subset \operatorname{sp}(u) \cap \operatorname{supp}(1-\tilde{\varphi}) \\
& \subset(\Lambda \cup \operatorname{sp}(f)) \cap \operatorname{supp}(1-\tilde{\varphi}) \\
& \subset \operatorname{sp}(f) .
\end{aligned}
$$

Remark 3.5. The above result is an extension of the classical one due to Massera (see $[7,9]$ for more information in this direction).

Recall that $f$ is quasiperiodic if it is of the form $f(t)=F(t, t, \ldots, t), t \in \mathbb{R}$, where $F\left(t_{1}, t_{2}, \ldots, t_{n}\right)$ is $\mathbb{X}$-valued continuous function of $n$ variables which is periodic on each variable. By [6, page 48], if $f$ is quasiperiodic and $\operatorname{sp}(f)$ is discrete, then $\operatorname{sp}(f)$ has an integer and finite basis, that is, there exists a finite subset $T \subset \operatorname{sp}(f)$ such that any element $\lambda \in \operatorname{sp}(f)$ can be represented in the form $\lambda=n_{1} b_{1}+\cdots+n_{m} b_{m}$, where $n_{j} \in \mathbb{Z}, b_{j} \in$ $T, j=1, \ldots, m$. Conversely, if $f$ is almost periodic and $\operatorname{sp}(f)$ has an integer and finite basis, $f$ is quasiperiodic. Finally, from Proposition 2.1(viii), we get immediately the following corollary.

Corollary 3.6. Let all assumptions of Theorem 3.4 be satisfied. Moreover, assume that $\mathbb{X}$ does not contain $c_{0}$. Then

(i) if $\operatorname{sp}(f)$ is countable, (1.1) has an almost periodic mild solution $w$ such that $\mathrm{sp}(w) \subset$ $\operatorname{sp}(f)$;

(ii) if $\mathrm{sp}(f)$ has an integer and finite basis, (1.1) has a quasiperiodic mild solution $w$ such that $\operatorname{sp}(w) \subset \operatorname{sp}(f)$.

Proof. (i) The theorem follows immediately from Theorem 3.4 and Proposition 2.1(viii).

(ii) By (i), we have that $w$ is almost periodic and $\operatorname{sp}(w) \subset \operatorname{sp}(f)$. Therefore, $\operatorname{sp}(w)$ has an integer and finite basis. Hence $w$ is quasiperiodic.

Using the estimate of the AP-spectrum of bounded mild solutions, we can improve the above corollary as follows.

Corollary 3.7. Let all assumptions of Theorem 3.4 be satisfied. Moreover, assume that $\mathbb{X}$ does not contain $c_{0}, \Lambda \cap \operatorname{sp}(f)$ is countable, and $f \in \mathrm{AP}(\mathbb{X})$. Then, (1.1) has an almost periodic mild solution $w$ such that $\mathrm{sp}(w) \subset \mathrm{sp}(f)$.

Proof. Choose $\varphi \in L^{1}(\mathbb{R})$ as in the proof of Theorem 3.4 and set $w=u-\varphi * u$. In Theorem 3.4, we have proved that $w$ is a mild solution of (1.1) such that (3.36) holds. We now show that $w \in \mathrm{AP}(\mathbb{X})$. In fact, by Proposition 2.4(iii) and Lemma 3.3(ii), we get

$$
\begin{aligned}
\operatorname{sp}_{\mathrm{AP}}(w) & =\operatorname{sp}_{\mathrm{AP}}(u-\varphi * u) \\
& \subset \operatorname{sp}_{\mathrm{AP}}(u) \cap \operatorname{supp}(1-\tilde{\varphi}) \\
& \subset \Lambda \cap \operatorname{supp}(1-\tilde{\varphi}) \\
& \subset \Lambda \cap \operatorname{sp}(f) .
\end{aligned}
$$


Since $\Lambda \cap \operatorname{sp}(f)$ is countable and $\mathbb{X}$ does not contain $c_{0}$, by Propositon 2.4(v), we have that $w$ is almost periodic.

To illustrate the usefulness of the above criterion for the almost periodicity of mild solutions of higher-order delay or advance abstract functional differential equations, we will consider the following examples.

Example 3.8. We consider a simple ordinary differential equation of the form

$$
\dot{x}(t)=f(t), \quad x(t) \in \mathbb{C}^{n},
$$

where $f$ is a $\mathbb{C}^{n}$-valued continuous $2 \pi$-periodic function. Obviously, the existence of a $2 \pi$-periodic solution to $(3.40)$ is equivalent to the $2 \pi$-periodicity of the primitive

$$
F(t)=\int_{0}^{t} f(\xi) d \xi, \quad t \in \mathbb{R}
$$

By an elementary argument, we can show that $F(t)$ can be represented in the form

$$
F(t)=m t+G(t)
$$

where $G$ is a continuous $2 \pi$-periodic function and $m=(1 / 2 \pi) \int_{0}^{2 \pi} f(\xi) d \xi$. Hence, for $F$ to be $2 \pi$-periodic, it is necessary and sufficient that $F$ be bounded. It is easy to see that under this condition, there is exactly one solution $x$ of (3.40) such that $\operatorname{sp}(x) \subset \operatorname{sp}(f)$.

This justifies the essential of our condition on the existence of a bounded mild solution in Theorem 3.4.

Example 3.9. In this example, we consider the oscillation equation

$$
\ddot{x}(t)+x(t)=\sin (1-\epsilon) t, \quad x(t) \in \mathbb{R},
$$

where $0 \leq \epsilon<1$ is a parameter. If $0<\epsilon<1$, the equation has the general solution that is of the form

$$
x(t)=C_{1} \cos t+C_{2} \sin t+\frac{1}{1-(1-\epsilon)^{2}} \sin (1-\epsilon) t
$$

where $C_{1}, C_{2}$ are two constants. It is clear that every solution is almost periodic. In this case, since there is no resonance, there exists only one almost periodic solution, that is,

$$
x_{1}(t)=\frac{1}{1-(1-\epsilon)^{2}} \sin (1-\epsilon) t
$$

with the same frequencies as the forcing term $f(t)=\sin (1-\epsilon) t$. 
Obviously, if $\epsilon=0$, the general solution of this equation is of the form

$$
x(t)=C_{1} \cos t+C_{2} \sin t-\frac{t}{2} \cos t
$$

where $C_{1}, C_{2}$ are constants. Therefore, since every solution of (3.43) in this case is unbounded, it has no $2 \pi$-periodic solutions.

Example 3.10. Consider the equation

$$
\begin{gathered}
u_{j t}(t, x)=d_{j} \Delta u_{j}(t, x)+\sum_{k=1}^{n} \int_{0}^{\infty} d b_{j k}(\tau) u_{k}(t-\tau, x)+f_{j}(x, t), \quad t \in \mathbb{R}, x \in \Omega, \\
d_{j} \frac{\partial u_{j}}{\partial v}(t, x)=0, \quad x \in \partial \Omega, j=1, \ldots, n,
\end{gathered}
$$

where $u(x, t), f(x, t)$ are scalar-valued functions, $d_{j} \geq 0, b_{j k} \in \mathrm{BV}\left(\mathbb{R}_{+}\right)$, for all $j, k=1, \ldots, n$, $\Omega \subset \mathbb{R}^{n}$ denotes a bounded domain with smooth boundary, and $\nu(x)$ the outer normal at $x \in \partial \Omega$.

This equation without the forcing term $f_{j}(t, x)$ has been investigated in [10, Section 6]. We define the space $\mathbb{X}:=\left[L^{2}(\Omega)\right]^{n}$ and $A: \mathbb{X} \rightarrow \mathbb{X}$ by the formula

$$
\begin{aligned}
A & =\left(\operatorname{diag} d_{j}\right) \Delta, \\
D(A) & =\left\{u \in \mathbb{X}: d_{j} u_{j} \in W^{2,2}(\Omega), d_{j} \frac{\partial u_{j}}{\partial \nu}(t, x)=0 \text { on } \partial \Omega\right\} .
\end{aligned}
$$

We define $B(t): \mathbb{X} \rightarrow \mathbb{X}$ by the formula

$$
(B(t) u)_{j}(x)=\sum_{k=1}^{n} b_{j k}(t) u_{k}(x), \quad u \in \mathbb{X}
$$

Then, (3.47) can be rewritten in the form

$$
\frac{d u(t)}{d t}=A u(t)+\int_{0}^{\infty} B(\tau) u(t-\tau)+f(t), \quad u(t), f(t) \in \mathbb{X}
$$

Let $u$ be a bounded uniformly continuous solution of (3.50) and let $f$ be an almost periodic function. Let $\mu_{0}=0>\mu_{1} \geq \mu_{2} \geq \cdots$ denote the eigenvalues of the Laplacian with Neumann boundary condition and $e_{j}$ the corresponding eigenvectors. By [10, Section 6], the imaginary spectrum of (3.50) is yielded as follows:

$$
\Lambda=\left\{\lambda \in \mathbb{R}: \chi_{m}(i \lambda)=0 \text { for some } m \in \mathbb{N}_{0}\right\}
$$

where

$$
\chi_{m}(i \lambda):=\operatorname{det}\left(\lambda-\mu_{m} D-\widehat{d B}(\lambda)\right), \quad m \in \mathbb{N}_{0}
$$


Moreover, one can prove that $\Lambda$ has Lebesgue measure zero. However, from this and the spectral inclusion in Lemma 3.3(ii)

$$
\operatorname{sp}_{\mathrm{AP}}(u) \subset \Lambda,
$$

we cannot conclude that $u$ is almost periodic. But if we set up more conditions on $f$ such that $\Lambda \cap \operatorname{sp}(f)$ is countable, then according to Corollary 3.7, there exists an almost periodic solution $w$ of equation (3.50) such that (3.36) holds.

\section{Acknowledgments}

The authors are grateful to the anonymous referee for the suggestion to improve the previous version of the paper. All correspondence should be addressed to the first author Nguyen Van Minh.

\section{References}

[1] W. Arendt and C. J. K. Batty, Almost periodic solutions of first-and second-order Cauchy problems, J. Differential Equations 137 (1997), no. 2, 363-383.

[2] __ Asymptotically almost periodic solutions of inhomogeneous Cauchy problems on the halfline, Bull. London Math. Soc. 31 (1999), no. 3, 291-304.

[3] W. Arendt and S. Schweiker, Discrete spectrum and almost periodicity, Taiwanese J. Math. 3 (1999), no. 4, 475-490.

[4] S. N. Chow and J. K. Hale, Strongly limit-compact maps, Funkcial. Ekvac. 17 (1974), 31-38.

[5] Y. Hino, T. Naito, N. Van Minh, and J. S. Shin, Almost Periodic Solutions of Differential Equations in Banach Spaces, Stability and Control: Theory, Methods and Applications, vol. 15, Taylor \& Francis, London, 2002.

[6] B. M. Levitan and V. V. Zhikov, Almost-Periodic Functions and Differential Equations, Moskov. Gos. Univ., Moscow, 1978, English translation by Cambridge University Press, Cambridge, 1982.

[7] J. L. Massera, The existence of periodic solutions of systems of differential equations, Duke Math. J. 17 (1950), 457-475.

[8] S. Murakami, T. Naito, and N. Van Minh, Evolution semigroups and sums of commuting operators: a new approach to the admissibility theory of function spaces, J. Differential Equations 164 (2000), no. 2, 240-285.

[9] T. Naito, N. Van Minh, and J. S. Shin, New spectral criteria for almost periodic solutions of evolution equations, Studia Math. 145 (2001), no. 2, 97-111.

[10] J. Prüss, Bounded solutions of Volterra equations, SIAM J. Math. Anal. 19 (1988), no. 1, 133149.

[11] Evolutionary Integral Equations and Applications, Monographs in Mathematics, vol. 87, Birkhäuser Verlag, Basel, 1993.

[12] W. M. Ruess, Almost periodicity properties of solutions to the nonlinear Cauchy problem in Banach spaces, Semigroup Theory and Evolution Equations (Delft, 1989), Lecture Notes in Pure and Appl. Math., vol. 135, Dekker, New York, 1991, pp. 421-440.

[13] W. M. Ruess and W. H. Summers, Weak almost periodicity and the strong ergodic limit theorem for periodic evolution systems, J. Funct. Anal. 94 (1990), no. 1, 177-195.

[14] Almost periodicity and stability for solutions to functional-differential equations with infinite delay, Differential Integral Equations 9 (1996), no. 6, 1225-1252.

[15] W. M. Ruess and Q. P. Vũ, Asymptotically almost periodic solutions of evolution equations in Banach spaces, J. Differential Equations 122 (1995), no. 2, 282-301. 


\section{Massera-type criterion}

[16] J. S. Shin and T. Naito, Semi-Fredholm operators and periodic solutions for linear functionaldifferential equations, J. Differential Equations 153 (1999), no. 2, 407-441.

[17] Q. P. Vũ, Almost periodic solutions of Volterra equations, Differential Integral Equations 7 (1994), no. 3-4, 1083-1093.

Nguyen Van Minh: Department of Mathematics, State University of West Georgia, Carrollton, GA 30118, USA

E-mail address: vnguyen@westga.edu

Ha Binh Minh: Department of Applied Mathematics, Hanoi University of Technology, Khoa Toan, Dai Hoc Bach Khoa Hanoi, 1 Dai Co Viet Road, Hanoi, Vietnam 


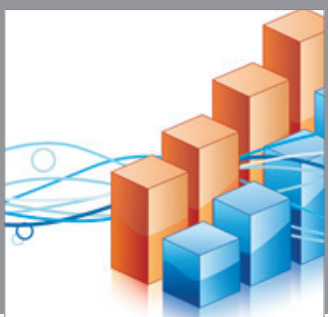

Advances in

Operations Research

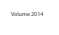

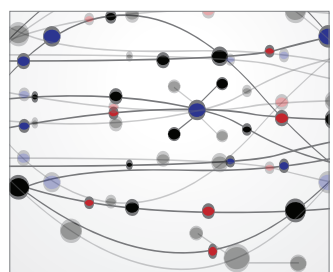

\section{The Scientific} World Journal
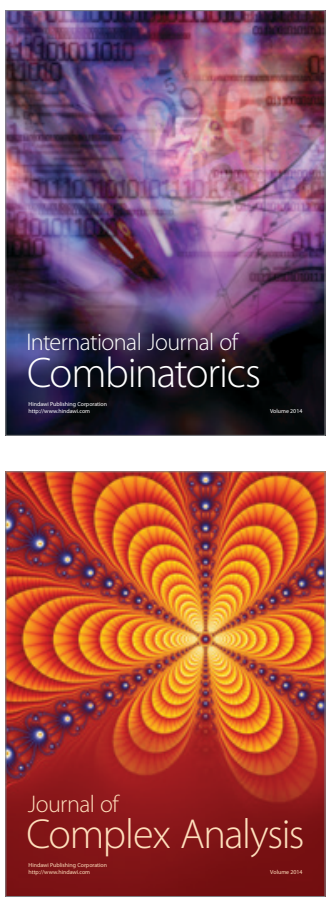

International Journal of

Mathematics and

Mathematical

Sciences
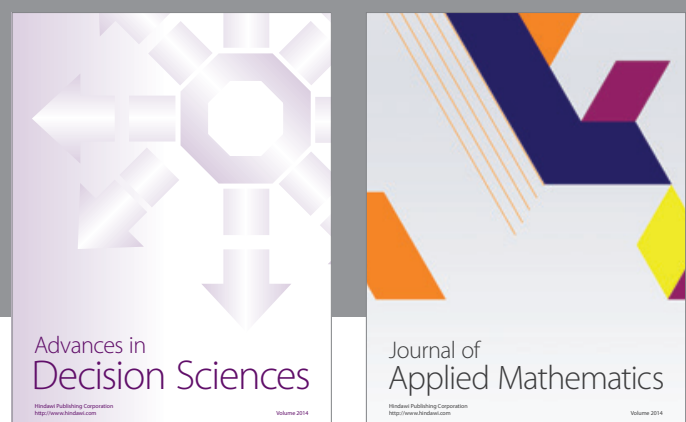

Journal of

Applied Mathematics
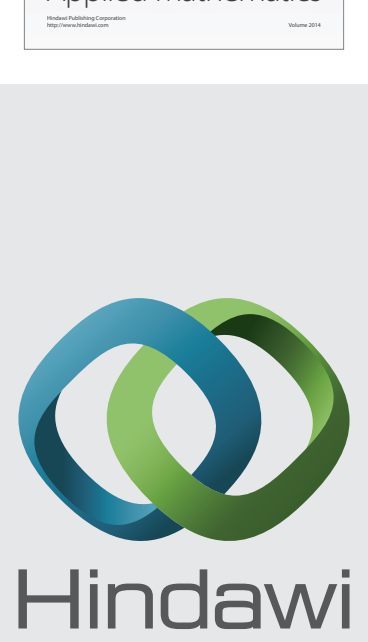

Submit your manuscripts at http://www.hindawi.com
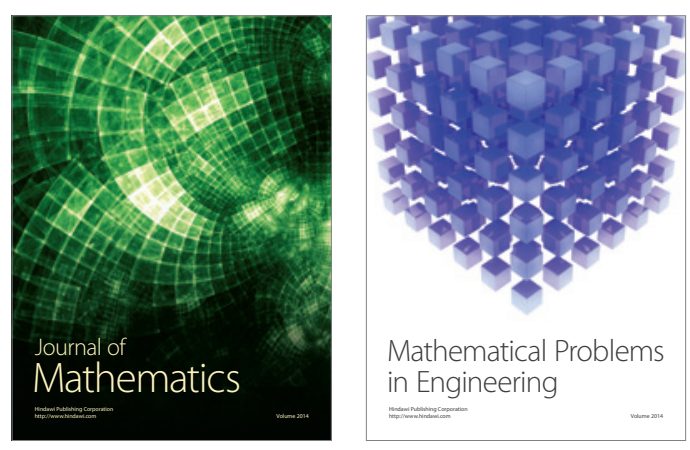

Mathematical Problems in Engineering
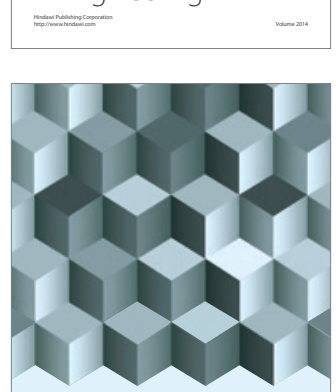

Journal of

Function Spaces
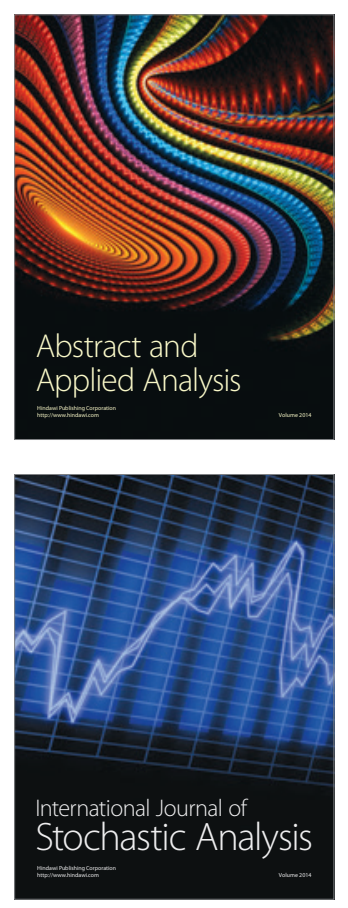

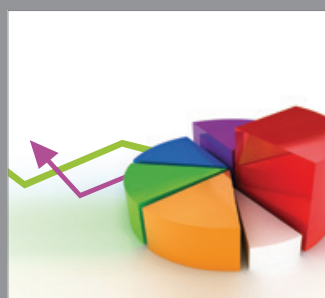

ournal of

Probability and Statistics

Promensencen
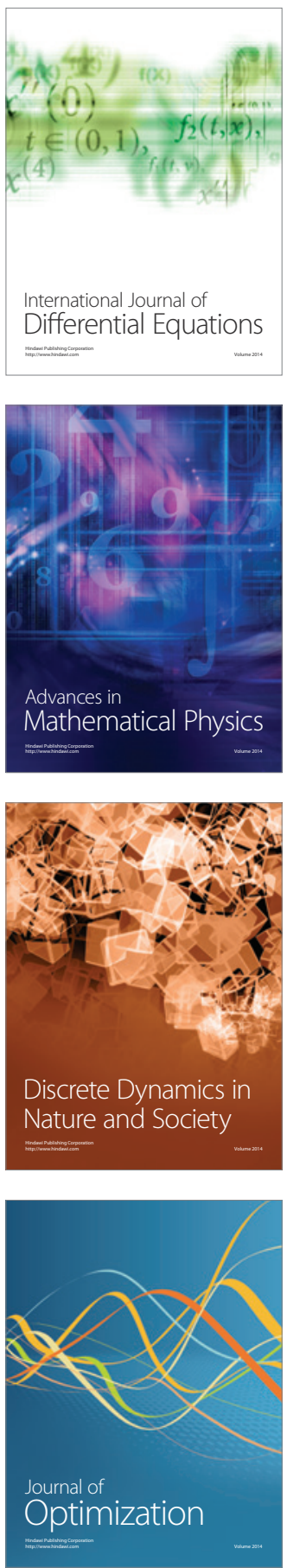